\title{
Experimental Organism Astrocytosis
}

National Cancer Institute

\section{Source}

National Cancer Institute. Experimental Organism Astrocytosis. NCI Thesaurus. Code C120865.

Reactive astrocytic proliferation often associated with degenerative, inflammatory or neoplastic changes in the central nervous system. 